\title{
Wendelin Foerster zum Gedächtnis.
}

Im und am grofsen Kriege starb schon bald (in der Nacht zum 18. Mai 1915). Wendelin Foerster. Auch ihn beklagen wir und beklagt besonders die Zeitschrift, der er jahrelang so manche seiner vortrefflichen Abhandlungen zuwandte, als eines seiner ungezählten Opfer. Der Ausbruch des Krieges zwang ihn Ägypten fern za bleiben, wo er im Winter 1914/15 einen letzten Versuch machen wollte, Linderung für sein Leiden und Kraft zu letüter Arbeit zu finden. So schrieb er mir denn am 6. Januar 1915: "Ich merke gerade jetzt bei dem elenden Wetter, wie mein Zustand sich schnell verschlimmert. Das war swar jedes Jahr so, aber dann flüchtete ich stets nach dem Süden ... So werde auch ich als Opfer des Krieges fallen." Auch erkannte Foersters Scharfblick bald, wie schwer uns mit unsern Bundesgenossen der Kampf sein würde, und es krampfte sich sein warmes deutsches Herz ob dieser deutschen Not. "Ich komme nicht voran und kann in meiner jetzigen Stimmung nicht arbeiten; gar heute wieder die Nachrichten aus dem Osten; auch im Westen sind wir eingefroren", schrieb er am 29. Oktober 1914. Er litt furchtbar unter dem unerhörten Treubruch Italiens, eines Landes, das ihm besonders teuer gewesen war und in dem er so oft Erholung gefunden hatte. Foersters schriftliche Auulserungen hierüber will ich lieber unterdrücken. Nur klein ist der Trost, dafs ihm, dem kernhaften Deutschböhmen, der innen und aufsen, bewafst und unbewufst gute deutsche Art darstellte, wenigstens das Schlimmste $2 u$ sehen erspart blieb. Doch an uns, die wir hoffen, uns wieder ungestörter Arbeit hingeben zu können, um schliefslich doch zu erreichen, wofür unsere Toten gestritten haben: die Geltung und Anerkennung deutschen Rechts und deutscher Art, ist es jetzt 1, eines unserer grolsen Forscher und Führer in Treue zu gedenken und uns an seinem Beispiel aufzurichten, um so wie er das Werk unseres grofsen Diez unentwegt fortzuführen.

Wendelin Foerster, ein Sohn des Riesengebirges, wurde 1844 am 10. Februar zu Wildschütz bei Trautenau im österreichischen

1 Verachiedene mifaliche Umstände, insbesondere meine Einziehung zum Heeresdienst, haben das Erscheinen dieses Nachrufs verzögert.

Zeitschr. f. rom Phil, XL 
Deutschlöhmen geboren. Er besuchte die Gymnasien in Leitomischel und Königgrätz und vollendete $(1861-65)$ den theologischen Lehrgang im bischöflichen Alumnat zu Königgrätz. Kurz vor den Weihen gab er die Theologie auf, fa/ste die Gymnasiallaufbahn ins Auge und studierte 1865-68 klassische Philologie in Wien, wo Bonitz und Vahlen seine Lehrer waren. Nach Abschlufs dieser Studien wird er zunächst Hilfslehrer am akademischen Gymnasium zu Wien und ist dann am deutschen Gymnasium in Brünn und darauf wieder in Wien am Josephstādtischen Gymnasium tätig. Zwischendurch erfolgen Foersters Umwandlung zum Romanisten und seine ersten Reisen zu Büchereien der Schweiz, Italiens und Frankreichs, wo zahlreiche Abschriften genommen werden. Verhältnismälsig spät erfolgt Foersters Promotion zum Doktor in der klassischen Philologie 1872 und Ostern 1874 die Habilitation für romanische Philologie in Wien.

Welche Fülle von Arbeit hatte der 30 jährige jetzt am Anfange seiner eigentlichen Laufbahn schon geleistet! Die Theologie und die klassische Philologie bewältigt, die junge Wissenschaft der Romanistik trotz der Gymnasiallehrertätigkeit und fast ohne Mittel erkämpft und erarbeitet, seine Handschriftenlade wohlgefültt! Dafs Foerster sich der klassischen Philologie entfremdete, lag besonders daran, dafs in ihr die Linguistik und die geschichtliche Sprachentwicklung zu kurz kamen. Dafür wäre Bonitz nicht $\mathrm{zu}$ haben gewesen und daher Foerster nicht länger für die klassische Philologie, der er jedenfalls seine beste Schulung verdankt. Nicht ohne Reiz ist in diesem Zusammenhang und bedauerlich zugleich, dafs Foerster als Romanist doch nie grölsere Arbeiten über lateinische Sprachgeschichte geliefert hat. Wohl schrieb er im Rheinischen Museum von 1878 über die Bestimmung der lateinischen Vokalquantität in positionslangen Silben aus dem Romanischen, sprach begeistert von Plautus, hielt vulgärlateinische Übungen, setzte sich für die richtige Aussprache des Lateinischen in den Schulen ein und kündigte wenigstens die Herausgabe von ${ }_{n}$ Anthimus und andern lateinisch-romanischen Texten für Seminarübungen" an. Er wollte auch nicht, dafs seine Schüler sich zu sehr von dem eigentlichen, neuen und reichen Arbeitsfeld der Romanisten abwandten, doch ist Emil Seelmanns bedeutendes Werk ${ }_{n}$ Aussprache des Latein nach physiologisch-historischen Grundsätzen ", Heilbronn 1885, mit Widmung an Foerster, der die Anregung gab, hier zu nennen.

Von der klassischen Philologie unbefriedigt gelassen wollte sich Foerster übrigens erst der slavischen zuwenden, zumal er von Jugend auf das Tschechische kannte und vermutlich auch schon damals das Polnische. Doch wurde er darauf hingewiesen, dafs man ihm, dessen Heimat so sehr im Kampfe gegen das Tschechentum gestanden habe, eine solche Wahl verargen müsse, und so ging es denn mit vollen Segeln hinüber zur jungen Wissenschaft unseres Diez. Der Erfolg blieb nicht aus. Schon nach einem 
halben Jahre (Herbst 1874) wurde Foerster als aufserordentlicher Professor auf den neugegründeten romanistischen Lehrstuhl nach Prag berufen, wurde 1876 daselbst Ordinarius und im Herbst desselben Jahres Diez' Nachfolger in Bonn. Hiermit beginnt die rastlose und erstaunlich fruchtbare Tätigkeit in der gesegneten Rheinstadt, die ein 32 jähriger begann und ein 71 jähriger beschlofs; sein letzter Arbeitstag war auch sein letzter Lebenstag, 17. Mai 1915, und in der ihm folgenden Nacht verschied der Unermüdliche im Schlaf sanft am Herzschlage. Wie andere, erfuhr auch ich seinen Tod rein zufällig durch eine Kölner Zeitungsnotiz, wie denn auch die sterblichen Reste des grofsen Toten in der Frühe und Stille des Morgens ohne irgend welche akademische Begleitung zur Einäscherung nach Mainz überführt wurden.

Das meiste an Foersters Leben ist Mühe und Arbeit, aber auch Erfolg gewesen. Sein Wollen und Vollbringen, sein klarer Plan und sein ganzes Einsetzen der Person ist wie bei dem ersten Napoleon. Aber nicht ist Foerster der selbstsüchtige Blender; ihm genügen anspruchslose Lebensbedingungen, und der ganze übrige Einsatz ist für die Sache selbst, die Wissenschaft. Für sie arbeitete er nach bester deutscher Art selbstlos und treu. Mancherlei Härten des Lebens, darunter schwere, zuletzt dauernde Krankheit vermochten seine Arbeitslust nicht zu erschüttern. Mehrfache Blinddarmentzündung war zu bestehen, chronische Bronchitis, Katarrhe, nervöse Herzschmerzen und schliefslich ein schweres Emphysem nötigen ihm immer wieder Klagen ab über die nalskalten Bonner Winter und vertreiben ihn nach dem Süden, und schon sechs Jahre vor seinem Tode, am 20. Juni 1909, schreibt er: „Ob ich jemals noch zum arbeiten komme, ist sehr unsicher, ich bezweifle es sehr ${ }^{u}$. Und doch war Foerster kurz vor seinem Tode noch gesonnen, "Alexius", "Roland" oder "Gral" zu vollenden und hatte ärziliche Auskunft darüber eingebolt, wie lange ihn der Tod noch freigäbe. Leider war es ihm nur bei dem ersten vergönnt, ihn in einer meisterlichen Studie in schärfste Beleuchtung zu rücken und die Textausgabe im Rohbau fertig zu stellen.

Erholung gönnte Förster sich wenig. Er kegelte in der Bonner Lese und war ein begeisterter Jäger, wobei er durch witzige Reden und Reime die Jagdgesellschaft aufs beste $\mathrm{zu}$ belustigen verstand. $\mathrm{Da}$ ls er mit Studenten jung sein konnte, bewies er uns auf einem Ausflug zum „Ännchen“ nach Godesberg, zu dem er sein Seminar und den neuphilologischen Verein eingeladen hatte. Er unterhielt wenig Verkehr und brach ihn seit 1908 mit Nah und Fern ab, was nicht hinderte, dafs er zu seinem 70 . Geburtstage, den er 1914 in San Remo beging, über 100 Glückwünsche erhielt. Sein eigentliches Reich bildeten seine beiden Arbeitszimmer, in die man durch gepolsterte Doppeltüren eintrat und an deren bücherreichen Wänden nur noch die Bilder von Diez und Bismarck einen Platz gefunden hatten. Wie wenige geizte Foerster mit seiner Zeit. Kam er in fremde Universitätsstädte, so suchte er seine Fachkollegen im all- 
gemeinen nicht auf, da er bei ihnen ebensowenig freie Zeit voraussetzte, wie er selbst hatte. Leider hielt Foersters schier unverwüstliche Kraft nicht gleichen Schritt mit seiner Regsamkeit und Frische. Er mufste öfter ein Semester oder länger aussetzen, und 1899 widmete er den 4. Band seiner grolsen Kristianausgabe neben Heinrich Zimmer drei Medizinern als ${ }_{n}$ seinen erfolgreichen Mitarbeitern ${ }^{\text {. }}$. Besonders spä̀ter, als er (seit 1908) vom Abhalten von Vorlesungen entbunden war, war es hart für den Unermüdlichen, in seiner Schaffenskraft geschwächt $\mathrm{zu}$ sein und nur wenige Stunden arbeiten zu können.

Wo Foerster einsetzte, arbeitete er schnell und erfolgreich, dazu half seiné erstaunliche Geistesschärfe und sein erstaunliches Gedächtnis. Über seine im Vergleich zu Gaston Paris hypothesenfeindliche Art, auch seine Beziehungen zu diesem, spricht er in der selbstbiographischen Auslassung in der grolsen Ausgabe des Karrenritters S. LXXXVIIff. Viel hat Foerster geschaffen und vollendet und noch mehr angekündigt. Er hätte einen Stab von Mitarbeitern haben müssen, um alles bereitliegende Material und alle angefangenen Arbeiten 20 bewältigen. Nun batte ja auch die Romanistik eine gewaltige Ausdehnung gewonnen, und die Lehrtätigkeit wurde stetig gröłser, so wie die Zahl der neusprachlichen Studenten wuchs, wozu 'dann die Studentinnen kamen, deren Studium Foerster wenig hold war.

Jedenfalls war die Lehrtätigkeit Foersters Freude und Trost. Das wissen wir aus manchen seiner Aussprüche, besonders aus der Zeit, wo er sich genötigt sah, sie aufzugeben ${ }^{1}$, und das bewies seine aufgeräumte und strahlende Art im Kolleg, das mit Scherzen und launigen Einfällen gewürzt war, besonders anch mit Zwischenfragen an ahnungslose Neulinge in den vorderen Bänken, in die sich sonst nur gut gewappnete Examenskandidaten empfeblungshalber wagten. Dies und der fesselnde, ausdrucksvolle Vortrag, die lebensvolle, freimütige, gro/szügige, mannhafte, ja streitbare Persönlichkeit, die in kraftvollen und mitunter beifsenden Aufserungen durchbrach, hielten den Hörer unweigerlich in Bann. Auch der häufigen Exkurse sei gedacht, die teils in die bunte Zettelwelt der Kolleghefte Aufnahme gefunden hatten, teils, vom Augenblick eingegeben, die verschiedensten Fragen und Erlebnisse betrafen und den Hörer sprunghaft in die Jagdgründe des Kottenforstes, die Dörfer Piemonts oder das flohreiche Sardinien versetzten. Foersters starke, fesselnde Persönlichkeit machten ihn volkstümlich und söhnten manche mit der gelegentlichen Schroffheit, mit der er die Wahrheit sagte, aus, während andrerseits manche Fehde ungeschlichtet blieb. Von keinem Dozenten wurde aufserhalb des Kollegs mehr gesprochen

1 Er schrieb mir am 6. November 1908: „Sie glauben nicht, welch bittern Schmerz mir die Aufgabe des Lehramts bereitet " (bittern einmal und Lehrdreimal unterstrichen). 
und seine Vorlesungen ungern versäumt. Allem Kleinlichen abhold schenkte er denen, die ihm năherstanden, das grölste Vertrauen, und das empfand besonders angenehm und ehrend der Bücherwart des Seminars, den er frei walten liers.

Bei aller frohen Wissenschaftlichkeit wufste Foerster seine Vorlesungen mit Geschick für den Bedarf des künftigen Oberlehrers einzurichten. Sein Einführungskolleg und seine phonetischen Ubungen waren in dieser Hinsicht besonders wertvoll. Er unterrichtete bestens und unparteiisch über die Reformbewegung und gab bei jeder Gelegenheit eine Menge praktischer Anweisungen, wobei ihm zustatten kam, dafs auch er jahrelang des Bakelamts gewaltet hatte, wie er sich einmal ausdrückte. Wie wertvoll Foersters Ratschläge waren, erfuhr ich anläfslich einer halbjährigen Studienreise nach Frankreich, wo sich mir gleichzeitig die eingebenden Kenntnisse des Landes, wie sie Foerster besals, enthüllten. Erwähnt sei nur, wie er am 2. September 1907 uber die Gesinnung der Franzosen schrieb: $n: \ldots$ alle deutschfreundlich, d. $h$. im Herzen sind es alles (ausnahmslos) Franzosen! Vergessen Sie es nie! und mögen die Leute noch so liebenswürdig sein “, was übrigens, nebenbei bemerkt, durchaus kein blofser Tadel ist.

- Die rein wissenschaftliche Anregung, die von Foerster ausging, wird erwiesen durch die äufserst stattliche Zahl von Bonner Doktorschriften und bedeutende wissenschaftliche Leistungen seiner Schüler an höherer Schule und Universităt. Traurig ist es, wie manche besonders befähigte Schüler des Meisters eines frühen Todes gestorben sind. Ein Apfelstedt, Hetzer, Heinrich Schneegans und Rechnitz sind darunter.

Foersters eigene wissenschaftliche Arbeiten umfassen zunăchst sein Hauptwerk, die vierbāndige grolse kritische Ausgabe der Werke Kristians von Troyes, Halle $1884-99$, wozu fast das gesamte Material schon im Jahre $1872 / 73$ gesammelt worden war. Zum Lobe dieser Riesenarbeit ist kein Wort mehr zu verlieren. Den völligen $\mathrm{Ab}$ schlufs wird sie erst erhalten, wenn auch der ${ }_{\text {G Gral }}{ }^{4}$, den G. Baist in Händen hat, in aller Form vorliegen wird. Noch turz vor seinem Tode konnte Foerster, wenn auch fern der Heimat und sterbenskrank, seine Ansichten und sein Wissen von Kristian, dem seine Hauptlebensarbeit gegolten hatte, unter Einschlurs des "Gral ${ }^{\mu}$ in einer Gesamtdarstellung zusammenfassen, nachdem einmal das Gesamtwörterbuch zu Kristians Werken durch seinen Mitarbeiter kompiliert und redigiert worden war, so dafs es nur noch der endgültigen Durchsicht des Meisters harrte. Lieber hätte sich Foerster übrigens mit dem Volksepos befafst, und er hat es oft bedauert, dals ihm der grofse Meister der Hofepik, der ihn ein Menschenalter beschäftigte, die Zeit dazu und zu manchen anderen, besonders linguistischen Arbeiten entzog.

Zu nennen ist weiter Foersters 1879 gegründete Altfranzösische Bibliothek ( 15 Bändchen, darunter von Foerster Lyoner Yzopet und 
zwei Rolandbändchen), die 1889 zur Romaniscten Bibliothek erweitert wurde (weitere 21 Bändchen, darunter 7 von Foerster: aufser den kleinen Kristianausgaben Wistasse le Moine [zus. mit J. Trost], Walters von Atrecht Ille und Galeron und Einleitung und Wörterbuch zu Kristians sämtlichen Werken). 1

Foersters Wiener Dissertation vom Jahre 1872 ist betitelt ${ }_{n} \mathrm{De}$ Rufi Breviario eiusque codicibus." Sie wurde 1874 der Ausgabe von "Rufi Festi Breviarium" (Wien, Hölder) nochmals vorgedruckt. Im gleichen Jahre erschien der erste altfranzösische Text Richars li Biaus. Es folgen: Dialoge Gregoire lo Pape 1876; Aiol nebst Elie de Saint Gile (2 Bände) 1876-80; Chevalier as deus espees 1877; Mocedades del Cid (anonym), Bonn 1878; Galloitalische Predigten (Roman. Stud. IV) 1879; Venus la deesse 1880; Garniers Tragédies (= Bd. I-IV von $\mathrm{K}$. Vollmöllers Neudrucken) 1882-83; Le università italiane e $i$ più recenti piani di riforma 1883; Altfranzösisches Úbungsbuch (zus. mit E. Koschwitz) 1884; dazu Erstes Zusatzheft von Foerster 1886; Sermon Saint Bernart (Romanische Forschungen II) 1885; Meigret, Grammère 1888; Freundesbriefe von Friedrich Diez, Bonn 1894; Sulla questione dell' autenticità dei codici di Arborea 1905; Vou de Luques 1906; Reichenauer Glossen 1907; Mervelles de Rigomer, I. Band 1907; Sankt Alexius, Beiträge zur Textlritik des ältesten französischen Gedichts 1914: Mervelles de Rigomer, II. Band (zusammen mit H. Breuer) 1915.

Dazu kommen zahlreiche längere und kürzere Abhandlungen und Kritiken in den Fachzeitschriften, darunter der Zeitschrift für die Österreichischen Gymnasien, dem Rheinischen Museum, dem Archivio glottologico usf. Weniger bekannt sind wohl ein Vortrag, den Foerster vor einer örtlichen gelehrten Gesellschaft in Bagnères de Bigorre über "cuistre und Verwandtes" hielt (in den Veröffentlichungen dieser Gesellschaft ca. 1897 gedruckt), und eine Abhandlung unter dem Strich in der Frankfurter Zeitung vom 24. und 25. Mai 1907, betitelt "Cicero oder Kikero und Verwandtes, eine sprachliche Betrachtung".

Manches Material liels Foerster bei Lebzeiten seinen Schülern zur Bearbeitung oder Herausgabe zukommen, vier gröfsere Sachen (Katharinenleben, Jehan de Lanson, Jaufré und Guarniers Thomas Becket) wird der Schreiber dieser Zeilen herausgeben, während andere handschriftliche Schätze nun unberührt in der Lade ruhen, wobei $\mathrm{zu}$ wünschen wäre, dals sie, gerade jetzt in der Zeit der erschwerten Beziehungen, der deutschen Wissenschaft erhalten blieben. Was es ist, lassen die Ankündigungen auf den Heftdeckeln der Romanischen Bibliothek zum Teil erkennen. Foersters Bibliothek von 2912 Nummern wurde im Dezember 1917 in Bonn versteigert.

1 Die Sammlung wird von A. Hilka fortgeführt werden. 
Die wissenschaftlichen Arbeiten des Meisters betrafen und förderten vornehmlich die geschichtliche Lautlehre ${ }^{1}$, die Mundartenkunde (insbesondere Nordfranzösisch, Waldensisch, Piemontesisch und Sardisch) die Wortgeschichte, die Textkritik und Paläographie und die Literaturgeschichte, letztere besonders durch seine anregenden Untersuchungen über die Entstehung der Artusromane, wo aber manche Punkte noch endgültiger Aufklärung harren. Dazu brachte er dann die erstaunliche Masse von Texten für Forscher und Studenten heraus. Alles das läfst die Wahl des 32 jährigen zum Nachfolger von Diez als eine glückliche und gerechtfertigte erscheinen, des Altmeisters, in dessen Geist er pietätvoll ${ }^{2}$ aufs beste weitergearbeitet hat, überall die sicheren Grundlagen die Diez gelegt, erweiternd und ausgestaltend, an Musterausgaben das Handwerk lehrend und durch starke eindrucksvolle Lehrtätigkeit Diez weit übertreffend. $\mathrm{Dafs}$ Foerster manche $\mathrm{Zweige}$ der ausgedehnten und sich stets weitenden Wissenschaft, die ihn bei seiner vielseitigen Befähigung hätten locken können, wenig pflegen konnte, wird nach obigem besonders der verstehen, der weifs, eine wie zeitraubende Sache die Herausgabe von Texten nach vielen Handschriften ist. Das Zeug zum Bahnbrecher hatte Foerster wie wenige, und umsomehr ist die Treue zu rühmen, die ihn bei Kristian aushalten liefs. Ein Schrecken, bei dem er bald erlahmte, waren für ihn mechanische Arbeiten, bei denen der Geist untätig bleiben mulste, und auffallenderweise rechnete er dazu auch lexikographische Arbeiten, wo doch in der alten Sprache hier noch grundlegend zu arbeiten ist. Eines habe ich oft bedauert, das Foersters Wirken besonders im Auslande, wo doch nur wenige im Deutschen sicher sind, sicherlich Eintrag getan hat: er schrieb bei allem Schwung und aller Kraft des Ausdrucks, einen etwas überhasteten, periodenreichen, oft schwer verständlichen Stil, schwierig umsomehr, als ja beim blofsen Lesen jeder stimmliche Ausdruck und jede Geberde fehlt. Gerade er hätte seinen sonst glücklichen Stil auch nach dieser Seite aufs beste vervollkommnen können, wenn ihm nur an dem Formalen mehr gelegen hätte. In Wort und Schrift verriet eben auch bei ihm der Stil den Menschen. Jedenfalls war Foerster eine Hauptgestalt der deutschen Wissenschaft im romanischen Auslande, an dessen Büchereien insbesondere er als ausgezeichneter Paläograph geschätzt wurde (so z. B. von Delisle an der Bibliotheque Nationale, der ihn gern zur Datierung schwieriger Codices heranzog), und wo er Mitglied gelehrter Gesellschaften in Turin, Mailand, Palermo, Montpellier und Brüssel war, eine Ehre, die ihm meines Wissens in Deutschland nur von den Akademien in Göttingen und

1 Die altfranzösische Grammatik, im Entwurf niedergeschrieben, ist nie erschienen, wie ja ähnlich die Suchiers auch nur zu einem Torso gediehen ist.

2 Foersters Einführungskolleg enthielt eine eindrucksvolle Schilderung von Diez' Leben und Wirken, und am Todestage des Altmeisters pilgerte er mit den Studenten zu des Altmeisters Grab auf dem weihevollen alten Bonner Friedhof, um einen Kranz niederzulegen. 
H. BREUER, WEMDELIN FORRSTER ZUM GEDXCHTNIS.

München zuteil goworden war und im übrigen von der Gesellschaft zur Förderung deutscher Wissenschaft, Kunst und Literatur in Böbmen.

So war es kaum oine geringere Ehre, Foersters als Diez' Lehrstuhl zu besteigen. Beide haben gezeigt, was deutschen Schaffens Urgewalt und deutsche Treue und Hingabe zu freierkorener Tătigkeit vermag. Möge beider Andenken und Vorbild besonders in diesem Sinne in der Romanistik lebendig und wirksam bleiben!

Hermann Breuer. 